in schizophrenia The interpretation of proverbs has a long tradition in the assessment of abstract think ing in schizophrenia. Recent investigations have emphasized the involvement of fronto-subcortical circuitry in proverb comprehension, executive function and schizophrenic psychopathology. The present study aimed to investigate the nature of proverb comprehension deficits and their relation to executive control function and psychopathology in schizophrenia. Another aim was to determine the specificity of proverb comprehension impairment in schizophrenia patients relative to other psychiatric populations.

Methods: Patients diagnosed with schizophrenia $(n=22)$ or alcohol use disorder $(n=20)$ and matched healthy controls $(n=34)$ were assessed on a multiple-choice version of a proverb comprehension task and on standard measures of response inhibition, working memory, divided attention and cognitive flexibility.

Results: Schizophrenia patients chose less frequently the correct (abstract-meaningful) and more often the concretistic (both meaningless and meaningful) proverb interpret ations than alcohol-dependent patients and healthy controls. They also selected more abstractmeaningless response alternatives and showed impaired performance across all assessed executive domains relative to healthy subjects. Divided attention and working memory, in particular, were related to proverb comprehension in the schizophrenia group. Furthermore, negative symptoms were associated with the number of abstractmeaningful responses and positive symptoms with the number of concretistic responses in the proverbs task.

Conclusions: Taken together, schizophrenia patients showed a specific pattern of proverb comprehension impairments which was differentially related to executive control subdomains and positive/negative symptoms. These findings might contribute to the development of new treatments specifically targeting pragmatic language impairments which have been shown to adversely affect social functioning in schizophrenia patients.

References

[1] Thoma, P., Daum, I. (2006). Neurocognitive mechanisms of figurative language processing-Evidence from clinical dysfunctions. Neuroscience and Biobehavioral Reviews, 30(8): 1182-1205

\section{4 - ANGER AS AN EMOTION-SPECIFIC DEFICIT IN DUAL-CHANNEL EMOTION RECOGNITION IN HAN SCHIZOPHRENIC PATIENTS}

Huai-Hsuan Tseng $^{1}$, Sue-Huei Chen ${ }^{2}$, Yu-Lien Huang ${ }^{2}$, Shi-Kai Liu ${ }^{3}$ ${ }^{T}$ National Taiwan University Hosptial, Taipei, Taiwan; ${ }^{2}$ Department of Psychology, National Taiwan University, Taipei; ${ }^{3}$ Department of Psychiatry, Far Eastern Memorial Hospital, Taipei, Taiwan r88227005@ntu.edu.tw

Introduction: Patients with schizophrenia perform significantly worse than controls on almost all measures of each nonverbal channel. Anger and fear were shown to have much lower acceptable levels in Chinese culture using an emotion recognition task made of multiethnic models. As a result, the previous conclusions of fear as the most distinguishable emotional-specific deficit in schizophrenic patients might be obscured in Han population.

Methods: A pilot study was proceeded beforehand to establish an effective tool for evaluating nonverbal accuracy (the Diagnostic Analysis of Nonverbal Accuracy 2-Taiwan version, DANVA2-TW), and then followed by formal study comparing the differences among 26 schizophrenic patients and 39 normal controls. Five subtests from WAIS-III, structured interview and self-report symptom checking list were also given to each subject. Data were analyzed by correlation analyses, analyses of variance, t-tests and regression analyses.

Results: Schizophrenic patients displayed less accurate performance than the normal controls in negative emotions, and performed significantly worse than normal subjects in recognizing anger emotions within both facial expression and prosodic channels, and fear in the prosodic channel.

Conclusions: A Deficit in emotion recognition in schizophrenia is universal across race, culture and perception modalities. However, emotion-specific deficits might present in different culture backgrounds. Anger recognition is a potential emotional-specific deficit in the Han population. A parallel test made of pure Han faces and Mandarin ones could simulate the real environment of Han society and provide a more accurate and delicate measurement of emotion recognition deficits.

\section{References}

[1] Ekman P, Freisen WV, Ancoli S: Facial signs of emotional experience. J of Personality \& Social Psychology 39(1 sup 6) 1980.

[2] Chan CC, Wong R, Wang K, et al.: Emotion recognition in Chinese people with schizophrenia. Psychiatry Res 157:67-76.

[3] Edwa rds J, Jackson HJ, Pattison PE: Emotion recognition via facial expression and affective prosody in schizophrenia: a methodological review. Clinical Psychology Review 22:789-832, 2002.

[4] Nowicki, S., \& Duke, M.P. (1994). Individual differences in the nonverbal communication of affect: The Diagnostic Analysis of Nonverbal Accuracy Scale. Journal of Nonverbal Behavior.

[5] Schneider, F., Gur, R.C., Gur, R.E., \& Shtasel, D.L. (1995). Emotional proce ssing in schizophrenia: neurobehavioral probes in relation to psychopathology. Schizophrenia Research, 17(1), $67-75$.

\section{5 - ATTENTION NETWORK TEST (ANT) REVEALS GENDER-SPECIFIC ALTERATIONS OF EXECUTIVE FUNCTION IN SCHIZOPHRENIA}

Carsten Urbanek, Carolin Opgen-Rhein, Andres Neuhaus, Eric Hahn, Michael Dettling

Charité, University Medicine Berlin, CBF, Dept. of Psychiatry and Psychotherapy, Berlin, Germany

carsten.urbanek@charite.de

Introduction: The Attention Network Test (ANT) provides measures for three different components of visual attention: executive control (= conflict inhibition), orienting, and alerting. There is reasonable evidence that alterations of attention - mainly in the executive/conflict domain - are associated with susceptibility to psychiatric illness. Specific impairments may be a characteristic of a medical condition such as schizophrenia and thus shift our understanding from a neuropsychological endophenotype to a more precise genetic comprehension of this disorder.

Methods: 35 schizophrenic patients and 35 healthy controls (13 female and 22 male in both groups) were enroled in the study. The ANT was administered to all participants and rated individual responses for the three factors (alerting, orienting, and conflict) an $\mathrm{d}$ their respective ratios relative to mean reaction times. With regard to gender differences, group comparisons were performed for schizophrenic patients vs. healthy controls.

Results: Significant differences between patients and controls could be detected for mean reaction time (639 vs. $538 \mathrm{~ms}$ ) and for conflict ratio (0.158 vs 0.191$)$. The latter difference mainly resulted from gender-specific variances of the conflict network in opposite directions. Conclusions: Executive function as represented by the conflict network of visual attention of the ANT is affected in schizophrenia. We have detected unreported gender-specific differences between healthy controls and schizophrenic patients. Especially with respect to the conflict network the ANT offers a promising methodology to detect a neuropsychological endophenotype of schizophrenia.

\section{References}

[1] Fan, J., McCandliss, B.D., Sommer, T., Raz, A., \& Posner, M.I. (2002). Testing the efficiency and independence of attentional networks. Journal of Cognitive Neuroscience, 14(3), 340-7.

[2] Hoff, A.L. \& Kremen, W.S. (2002). Sex differences in neurocogni tive function in schizophrenia. In F. Lewis-Hall, T.S. Williams, J.A. Panetta, \& J.M. Herrera (Eds.), Psychiatric Illness in Women: Emerging Treatments and Research chapter 9, (pp. 215-238). American Psychiatric Publishing.

[3] Posner, M.I. \& Petersen, S.E. (1990). The attention system of the human brain. Annual Review of Neuroscience, 13, 25-42. 